DOI: https://doi.org/10.34069/AI/2022.49.01.26

How to Cite:

Popovych, I., Stepanenko, L., Savchuk, O., Zavatska, N., \& Koval, I. (2022). Estados mentales dominantes de los militares: parámetros, estructura, interdependencia de los factores. Amazonia Investiga, 11(49), 239-250.

https://doi.org/10.34069/AI/2022.49.01.26

\title{
Estados mentales dominantes de los militares: parámetros, estructura, interdependencia de los factores
}

\section{Servicemen's dominant mental states: parameters, structure, and factor interdependence}

Received: November 28, 2021

\section{Resumen}

El objetivo del artículo es fundamentar teóricamente y estudiar empíricamente los estados mentales dominantes de los militares que sirven en el ejército, algunos de los cuales participaron en operaciones militares; determinar los parámetros, estudiar la estructura y la interdependencia de los factores. Se utilizó las herramientas de psicodiagnóstico que reflejaron de manera relevante el sujeto y el objeto de la investigación. Se interpretó cualitativamente los estados mentales dominantes de los encuestados, se aclaró el número óptimo de factores de la investigación y se describió las características semánticas psicológicas del fenómeno estudiado. El análisis factorial ANOVA permitió determinar la estructura de los estados mentales dominantes de los encuestados, que combina siete estados actuales (F1-F7), lo que constituye el $71.651 \%$ de la dispersión. El más importante de la estructura factorial es F4 "La Dominación pragmática", que tiene más interrelaciones $(\mathrm{n}=$ 4) y la conexión más estable con $\mathrm{F} 1$ ( $\mathrm{rs}=.333$; $\mathrm{p} \leq .01$ ). Se argumentó que los parámetros
Accepted: January 15, 2022

Written by:

Ihor Popovych ${ }^{103}$

https://orcid.org/0000-0002-1663-111X

Larysa Stepanenko ${ }^{104}$

https://orcid.org/0000-0002-2891-9006

Oleksandr Savchuk ${ }^{105}$

https://orcid.org/0000-0002-8309-5927

Nataliia Zavatska ${ }^{106}$

https://orcid.org/0000-0001-8148-0998

Iryna Koval ${ }^{107}$

https://orcid.org/0000-0002-2788-6345

\footnotetext{
${ }^{103}$ Doctor of Psychological Sciences, Full Professor, Full Professor of the Department of Psychology, Kherson State University, Kherson, Ukraine.

104 Candidate of Psychological Sciences, Associate Professor, Department of Psychology, SHEI "Donbas State Pedagogical University", Sloviansk, Ukraine.

${ }^{105}$ Candidate of Psychological Sciences, Senior Researcher, Department of Philosophy, Ivan Kozhedub Kharkiv National University of Air Force, Kharkiv, Ukraine.

${ }^{106}$ Doctor of Psychological Sciences, Full Professor, Head of the Department of Practical Psychology and Social Work, Volodymyr Dahl East-Ukrainian National University, Severodonetsk, Ukraine.

${ }^{107}$ Doctor of Psychological Sciences, Associate Professor, Full Professor of Department of Medical Psychology, Psychosomatic Medicine and Psychotherapy, Bogomolets National Medical University, Kyiv, Ukraine.
} 
psicológicos, la estructura y la interdependencia de factores son componentes clave para resolver las tareas de actividad táctica, de servicio, de fuego y de combate. Es conveniente poner en práctica los resultados del estudio durante la formación profesional y el entrenamiento táctico de los militares.

Palabras clave: esfera de necesidad motivacional, motivo, psicología militar, estrés, actividad militar.

\section{Introducción}

Actualmente, en el mundo hay casi cuatro docenas de conflictos armados y guerras, una de las cuales continúa en el este de Ucrania. En particular, según los datos a principios de 2017 Center for Systemic Peace (Centro Estadounidense de la Paz Integral) (CSP) contó 36 guerras en las cuales participan 28 estados. Casi la mitad de ellos (13 países) tienen conflictos prolongados, que duran más de 10 años (Center for Systemic Peace, 2017). La militarización de la sociedad, los conflictos militares actuales en diferentes partes del mundo, en particular en el este de Ucrania, exigen que la comunidad científica sea responsable y empiece a actuar activamente. La comunidad científica, a través de la investigación, los hechos confiables, la opinión pública oportunamente formada y las medidas preventivas, puede salvar cientos de miles o aun millones de vidas humanas y unir la humanidad en torno de los objetivos pacíficos. La comprensión crítica integral de las cuestiones jurídicas internacionales, procesales así que las de carácter organizativo de la investigación de crímenes de guerra es igualmente importante (Arbeláez-Campillo, Dudareva \& Rojas-Bahamón, 2019; Tataryn et al., 2021).

\section{Revisión de la literatura}

El problema de la esfera de necesidad motivacional, las pretensiones del individuo son sumamente actuales en diversas áreas de la actividad humana: socioeconómica (Arbeláez-Campillo, Rojas-Bahamón, \& Arbeláez-Encarnación, 2019), (Pinkovetskaia et al, 2019), educativa y profesional (Blynova et al., 2020a), deportiva (Popovych et al., 2020a), competitiva Cheban (et al., 2020) y otras áreas con cargas excesivas que están al límite de capacidades humanas (Kuzikova et al., 2021; Nosov et al., 2020; Zinchenko et al., 2019; 2020). La esfera militar pertenece a las áreas extremas de la actividad humana, ya que va acompañada de importantes cargas psicoemocionales y físicas, situaciones extremas. Este tipo de should be operationalized in the professional education and tactical training of servicemen.

Key words: needs and motivational sphere, motive, military psychology, stress, military activity.

actividad adquiere un significado especial en el momento en que el país se encuentra en conflicto militar, lo que hace a todo el personal militar, incluidos reclutas y contratistas, estar en alerta máxima. Consideramos que este estado de mayor preparación es el estado mental dominante del militar que tenemos por objetivo de estudiar.

La actividad militar es una actividad profesional de una persona que está experimentando cambios socioculturales permanentes, desafíos actuales. La actividad profesional de los militares es extremadamente sensible al progreso técnico, las tecnologías digitales de la información y los procesos de comunicación de la sociedad. Está claro que la motivación no puede existir aislada de la actividad humana, por eso nuestro objetivo es investigar justamente en la actividad actual y determinar los parámetros, la estructura factorial y la interdependencia de los factores que dominan los estados mentales de militares. Se realizó un análisis retrospectivo de los parámetros esenciales psicológicos de la esfera motivacional de la personalidad. (Elers, 2002; Hekhauzen, 2003). Se tuvo en cuenta las herramientas de psicodiagnóstico necesarias para estudiar estos fenómenos. (Herbachevskyi, 1990). En algunas fuentes, los parámetros psicológicos esenciales de la motivación se describen como componentes estructurales que forman un fenómeno multifacético integrado (Hekhauzen, 2003). Esta interpretación provoca el aspecto multifacético del contenido categórico y conceptual del fenómeno estudiado y la inconcreción del sistema de conceptos psicológicos, a pesar de que encontramos la justificación de la lista significativa de parámetros esenciales psicológicos en los conceptos conocidos, generalmente en el contexto de la actividad profesional, la formación profesional y personal. (Hekhauzen, 2003). Por supuesto, los estados mentales dominantes son los parámetros esenciales psicológicos muy importantes para la esfera motivacional de una personalidad. Bajo el estado mental entendemos 


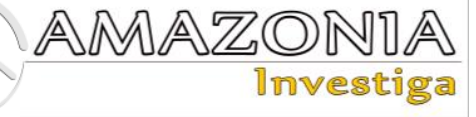

la peculiaridad de la actividad mental, debida a sus características semánticas y la actitud humana hacia esta actividad. Como los estados mentales son una integración estable de todas las manifestaciones mentales del hombre en su interacción determinada con la realidad, generalmente ellos se reflejan en la funcionalidad general de la psíquica. Nos vemos obligados a señalar que la dimensión empírica del estado mental dominante de los militares requiere una investigación cuidadosa en la actual actividad profesional militar. Precisamente en tales condiciones aspiramos a encontrar respuestas a las preguntas que ayudarán a comprender mejor la esencia de este fenómeno psicológico. Al construir la imagen empírica del estudio, tuvimos en consideración los resultados de una serie de trabajos sobre la problemática de actualidad. (Halian et al., 2020; 2021).

El estado mental es el nivel general funcional de la actividad mental, que depende de las condiciones de la actividad profesional, incluso de la inactividad y de sus características individuales psicológicas. Los estados mentales pueden ser de corta duración, situacionales, estables, personales y tener una serie de otras características de clasificación. (Prokhorov et al., 2015).

Creemos que el estado mental dominante es un tipo del estado mental que se integra con los procesos y propiedades mentales, y es capaz de actuar como un regulador importante del encuestado en cualquier actividad. Este estado está directamente relacionado con el éxito/fracaso del trabajo realizado. Como la organización eficaz de la actividad profesional de las unidades militares requiere del cuerpo de oficiales el conocimiento de la estructura y las particularidades esenciales psicológicos de los estados mentales dominantes de los militares. El desempeño de las misiones de servicio (de combate) por los militares depende directamente del tipo de estado mental dominante. La comprensión del cuerpo de oficiales de los dominantes de estados mentales permite operacionalizar la resolución de los objetivos de la preparación profesional al servicio militar y a la instrucción de combate. Los estados mentales dominantes acompañan el proceso de la actividad profesional y se puede presentarlos como interioridad, exterioridad, actividad, pasividad, apertura, reserva etc. (Popovych et al., 2021b). Según C. Izard (1991) los estados mentales están estrechamente relacionados con la actividad mental, a veces la necesidad de actuar toma la forma de estrés mental.
Se estableció que los estados mentales de expectativas influyen en el curso de los procesos mentales, y a menudo repitiéndose, adquieren estabilidad y se convierten en la propiedad del individuo. Los estados mentales tienen la capacidad de acordarse con las necesidades y aspiraciones del hombre, con sus capacidades y recursos, asegurando su desarrollo en las condiciones específicas del espacio (Popovych et al., 2021b).

Bajo el estado mental dominante del militar entendemos el conjunto integrado de las características disponibles que afectan el resultado de la actividad profesional, el desempeño de las tareas oficiales (de combate). Estos puntos de vista se confirman metodológicamente en el estudio de los estados cognitivos durante la actividad intelectual de los estudiantes a través de la estructura del estado de interés/estrés mental (Prokhorov et al., 2015), en los resultados del estudio del estado mental de expectativas en la actividad educativa profesional (Popovych et al., 2020a) y en los estudios del estado mental de fatiga crónica, que perjudica el rendimiento físico de una persona (Marcora et al, 2009) etc. Existe un estudio que descubre como el estado mental primario de las expectativas de un niño se convierte en un estado concreto, objetivo, definido, es decir, el estado mental de las expectativas de un adulto. (Popovych, 2014).

El análisis retrospectivo de fuentes científicas da motivos para generalizar que el lugar y el papel de los estados mentales dominantes de los militares que participaron en las operaciones militares o estaban preparados para tales acciones son poco estudiados.

A pesar de toda la importancia y actualidad, la descripción de las dimensiones teóricas y metodológicas del estudio de los estados mentales dominantes de militares sigue siendo un problema que requiere un análisis científico cuidadoso, una investigación empírica y la justificación.

Hipótesis. Suponemos que los resultados del estudio de los estados mentales motivacionales dominantes de militares tendrán datos científicos valiosos y será recomendable ponerlos en práctica en la formación profesional y entrenamiento táctico de los militares.

Propósito. Justificar teóricamente y estudiar empíricamente los estados mentales dominantes de militares y determinar los parámetros 
psicológicos, la estructura y la interdependencia de los factores.

\section{Método}

Los trabajos experimentales de los científicos A. Prokhorov y colegas (Prokhorov et al., 2015), V. Plokhikh y colegas (Plokhikh, 2021; Plokhikh et al., 2021) y I. Popovych y colegas (Popovych et al., 2020b) sobre los estados mentales constituyen la base metodológica de la investigación. Se tiene en cuenta que la parte predominante de los jovenes estudiados es de edad de 18 a 20 años $(n=54 ; 62.79 \%)$, es decir los que cumplían el servicio militar obligatorio. En consecuencia, durante la organización del estudio, se toma en consideración las reglas psicológicas de la adolescencia descritas y fundamentadas en los estudios de las cosmovisiones (Kononenko et al., 2020), las orientaciones valorativas (Popovych at 2021c; Shevchenko, 2019), las reglas de la actividad educativa profesional de jovenes (Blynova et al., 2020b; Shevchenko et al., 2020), el bienestar psicológico (Blynova \& Kruglov, 2019; Ma et al., 2020; Semenov et al., 2021), el maximalismo y perfeccionismo de jóvenes (Klenina, 2019), el uso abusivo de redes sociales (Hudimova, 2021; Hudimova et al., 2021), la regulación espacial del comportamiento (Khmiliar et al., 2020) y otros estudios relacionados (Popovych et al., 2021a).

Participantes. En el estudio de constatación participaron los militares de las Fuerzas Armadas de Ucrania, que hacen el servicio en la base de la unidad militar AXXXX (el nombre fue cambiado - I. P., L. S., O. S., N. Z., I. K.), que se disloca en el territorio de la región de Kherson, de 18 a 45 años, con diferente estado civil y situación social. Los militares tienen diferentes rangos, desde soldado hasta capitán, diferentes puestos, desde conductor hasta piloto-navegante. Diferente experiencia laboral: mantenimiento de la paz, participación en la Operación Antiterrorista (OA) en el Este de Ucrania, participación en la Operación de Fuerzas Unidas (OFU). El número total de estudiados es 86 personas $(67$ hombres, lo que constituye $77.91 \%$, y 19 mujeres, que forma $22.09 \%$ ); la edad media es 23,19 años. De todos los estudiados $(\mathrm{n}=54 ; 62,79 \%)$ hacían el servicio militar activo, el resto de los militares estaban bajo contrato $(\mathrm{n}=32 ; 37,21 \%)$. El estudio fue realizado de acuerdo con los principios éticos del Comité de los Derechos de Investigaciones experimentales de la Declaración de Helsinki (2013).

Procedimiento e instrumentos. Desde mediados de 2020 y durante todo el año 2021, se utilizó los métodos de prueba válidos con cuestionarios estandarizados. El método de diagnóstico motivacional de la personalidad para tener éxito (Elers, 2002) permitió medir el parámetro psicológico de la motivación para el éxito (ME). El índice de confiabilidad de $\alpha$-Cronbach fue $\alpha \mathrm{ME}=.914$. Con ayuda del cuestionario "Nivel de pretenciones de la personalidad" (NPP) (Herbachevskyi, 1990) midieron los parámetros psicológicos siguientes: motivación interna (MI), motivación cognitiva (MC), motivación de la evitación (ME), motivación de la competencia (MC), motivación del cambio de actividad (MCA), motivación de la autoestima (MA), relevancia de los resultados (RR), complejidad de tareas (CT), esfuerzo de voluntad (EV), evaluación del nivel de los resultados obtenidos (ENR), evaluación del potencial propio (EPP), nivel previsto de la movilización de esfuerzos (NPME), nivel esperado de los resultados (NER), regularidad de los resultados (RR), iniciativa (I). Las respuestas se evaluaron mediante la escala de diferencial semántico bipolar, cuyos valores oscilaron entre -3 (no estoy nada de acuerdo) y +3 (estoy absolutamente de acuerdo). Los índices de confiabilidad obtenidos mediante el estadístico de $\alpha$-Cronbach son: $\alpha$ Рдо $=.834$. Se utilizó el coping test "Way of Coping Questionare" ("WCQ"), elaborado par (Lazarus \& Folkman, 1984) y adaptado par T. Kriukova y Ye. Kuftiak (2007). El coping test permitió determinar ocho parámetros psicológicos - ocho métodos para superar las dificultades en el campo de las actividades militares actuales: confrontación (C), distanciamiento (D), autocontrol (AC), búsqueda del apoyo social (BAS), aceptación de la responsabilidad (AR), evitación (E), planificación de la resolución del problema (PRP), reevaluación positiva (RP). Las respuestas se evaluaron con ayuda de la escala de diferencial semántico unipolar, cuyos valores oscilaron entre 0 (nunca) y 3 (frecuentemente), $\alpha$-Cronbach es $\alpha_{W C Q}=.855$. Como se puede ver, los índices de la confiabilidad de los métodos aplicados y los tests de $\alpha$-Cronbach están entre el nivel medio (.8) y el nivel alto (.9).

Análisis estadístico. El procesamiento estadístico de los datos empíricos se realizó mediante el programa estadístico "SPSS" versión 24.0 y MS "Excel". Para determinar la interdependencia de los factores se usó los coeficientes de correlación de Spearman $\left(r_{s}\right)$. Para definir la estructura factorial se utilizó el análisis factorial ANOVA. Se determinó las características de frecuencia de los parámetros psicológicos. Consideramos que las diferencias son estadísticamente significativas en el nivel $\mathrm{p} \leq .05$. 


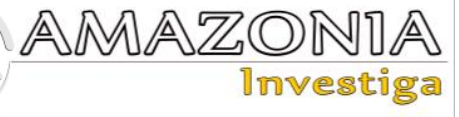

Resultados

Los resultados de las medidas empíricas fueron evaluados según todos los métodos, enfocándose en las escalas de la media aritmética (M) y la desviación cuadrática media (SD).

Tabla 1.

Características de frecuencia según el método de diagnóstico motivacional de la personalidad para tener éxito $(n=86)$

\begin{tabular}{lll}
\hline Escala & Media aritmética (M) & $\begin{array}{l}\text { Desviación cuadrática media } \\
\text { (SD) }\end{array}$ \\
\hline Motivación para el éxito (ME) & 16.87 & 3.62 \\
\hline
\end{tabular}

Fuente: Elaboración propia, 2021.

Los resultados obtenidos de la media aritmética y la desviación cuadrática media de la muestra $(\mathrm{M}=16.87 ; \mathrm{SD}=3.63)$ son similares a los datos obtenidos de otros estudios relevantes
En Tabl. 1 presentamos las características de frecuencia de las medidas según el método de diagnóstico motivacional de la personalidad para tener éxito (Elers, 2002).

Tabla 2.

Características de frecuencia del coping-test “WCQ” $(n=86)$

\begin{tabular}{lll}
\hline Escala & Media aritmética (M) & $\begin{array}{l}\text { Desviación cuadrática media } \\
\text { (SD) }\end{array}$ \\
\hline Confrontación (C) & 43.11 & 17.91 \\
Distanciamento (D) & 43.23 & 24.01 \\
Autocontrol (A) & 71.19 & 18.98 \\
Busqueda del apoyo social & 39.23 & 21.12 \\
(BAS) & & \\
Aceptación de la & 59.61 & 19.67 \\
responsabilidad (AR) & 38.04 & 21.01 \\
Evitación (E) & 69.98 & 17.44 \\
Planificación de la resolución & del problema (PRP) & 20.55 \\
Reevaluación positiva (RP) & 51.72 & \\
\hline
\end{tabular}

Fuente: Elaboración propia, 2021.

Los resultados obtenidos de media aritmética y la desviación cuadrática media de la muestra del coping-test "WCQ" son superiores que los de los encuestados jóvenes, obtenidos en otros estudios usando el mismo método, pero las diferencias no son significativas (Popovych, 2020a).
(Popovych, 2019), lo que confirma la validez de los datos obtenidos. En Tabl.2 presentamos las características de frecuencia de las medidas del coping-test "WCQ" (Lazarus \& Folkman, 1984).

Tabla 3.

Características de frecuencia según el cuestionario "NPP" ( $n=86)$

\begin{tabular}{|c|c|c|}
\hline Escala & Medida aritmética (M) & $\begin{array}{l}\text { Desviación cuadrática media } \\
\text { (SD) }\end{array}$ \\
\hline Motivación intrínseca (MI) & 13.23 & 3.38 \\
\hline Motivación cognitiva (MC) & 16.33 & 3.16 \\
\hline $\begin{array}{l}\text { Motivación de la evitación } \\
\text { (ME) }\end{array}$ & 12.24 & 3.87 \\
\hline $\begin{array}{l}\text { Motivación de la competencia } \\
\text { (MC) }\end{array}$ & 12.29 & 3.85 \\
\hline
\end{tabular}

Constatamos la fiabilidad de los datos obtenidos. En Tabl. 3 presentamos las características de frecuencia de las medidas según el cuestionario "Nivel de pretenciones de la personalidad" ("NPP") (Herbachevskyi, 1990). 


\begin{tabular}{|c|c|c|}
\hline $\begin{array}{l}\text { Motivación del cambio de } \\
\text { actividad (MCA) }\end{array}$ & 13.44 & 3.80 \\
\hline $\begin{array}{l}\text { Motivación de la autoestima } \\
\text { (MA) }\end{array}$ & 14.88 & 3.35 \\
\hline $\begin{array}{l}\text { Relevancia de los resultados } \\
\text { (RR) }\end{array}$ & 9.51 & 3.07 \\
\hline Complejidad de tareas (CT) & 6.17 & 2.99 \\
\hline Esfurzo de voluntad (EV) & 13.27 & 3.25 \\
\hline $\begin{array}{l}\text { Evaluación del nivel de los } \\
\text { resultados obtenidos (ENRO) }\end{array}$ & 10.75 & 2.35 \\
\hline $\begin{array}{l}\text { Evaluación del potencial propio } \\
\text { (EPP) }\end{array}$ & 14.64 & 3.29 \\
\hline $\begin{array}{l}\text { Nivel previsto de la } \\
\text { mobilización de esfuerzos } \\
\text { (NPME) }\end{array}$ & 15.08 & 2.89 \\
\hline $\begin{array}{l}\text { Nivel esperado de los resultados } \\
\text { (NER) }\end{array}$ & 10.19 & 2.37 \\
\hline $\begin{array}{l}\text { Regularidad de los resultados } \\
\text { (RR) }\end{array}$ & 13.95 & 3.04 \\
\hline Iniciativa (I) & 13.63 & 2.98 \\
\hline
\end{tabular}

Fuente: Elaboración propia, 2021.

Los resultados obtenidos de la media aritmética y la desviación cuadrática media de la muestra según el cuestionario "NPP" también son similares a los datos de otros estudios relacionados en los que se utilizó este método (Popovych, 2019; Popovych et al., 2021c). Constatamos la fiabilidad de las medidas obtenidas. El estudio de los estados mentales dominantes de los militares está representado por veinticuatro parámetros psicológicos. Consideramos apropiada tal elección, ya que los parámetros propuestos reflejan de manera relevante la actualidad de la situación simulada de elección así que la voluntad de los encuestados.

Usando el método del componente principal, se realizó la reducción de la proporcionalidad de los factores recomendados y se determinó su carga. Se reveló que siete factores tienen valores superiores a 1.0 y reflejan el $71.651 \%$ de la dispersión de las variables esbozadas (Tabl. 4).

Tabla 4.

Matriz de cargas factoriales

\begin{tabular}{|c|c|c|c|c|c|c|c|}
\hline Escala & F1 & F2 & F3 & F4 & F5 & F6 & F7 \\
\hline MTE & & & .724 & & & & \\
\hline MI & .524 & & & & & & \\
\hline $\mathrm{MC}$ & .577 & & & & & & \\
\hline ME & & & & & .520 & & \\
\hline $\mathrm{MC}$ & & & .749 & & & & \\
\hline MCA & & -.652 & & & & & \\
\hline MA & .483 & & & & & & \\
\hline $\mathrm{RR}$ & -.445 & & & & & & \\
\hline CT & & & & & & .606 & \\
\hline EV & .539 & & & & & & \\
\hline ENR & & & & & -.616 & & \\
\hline EPP & .622 & & & & & & \\
\hline NPME & .748 & & & & & & \\
\hline NER & & & .434 & & & & \\
\hline $\mathrm{RR}$ & .593 & & & & & & \\
\hline I & & .597 & & & & & \\
\hline $\mathrm{C}$ & -.547 & .564 & & & & & \\
\hline D & & & & -.585 & & & \\
\hline $\mathrm{AC}$ & -.520 & & & & & & \\
\hline BAS & & & & & & .584 & \\
\hline AR & & -.545 & & & & & .577 \\
\hline
\end{tabular}




\begin{tabular}{llllllll}
\hline E & & $\mathbf{. 5 9 8}$ & & $\mathbf{. 7 9 1}$ & & & \\
PRP & & $\mathbf{- . 5 5 6}$ & $\mathbf{- . 5 5 6}$ & & & & \\
RP & & 15.187 & 11.948 & 9.176 & 6.466 & 6.022 & 4.484 \\
Dispersión, \% & 18.368 & & & & \\
$\sum_{\%}^{\text {de dispersión, }}$ & 18.368 & 33.555 & 45.503 & 54.679 & 61.145 & 67.167 & 71.651 \\
Valor & 4.468 & 3.645 & 2.885 & 2.221 & 1.567 & 1.458 & 1.098 \\
\hline
\end{tabular}

Fuente: Elaboración propia, 2021.

Nota. Las variables significativas se destacan en negrita.

En la tabla podemos ver los parámetros que tienen una carga factorial inferior a .500 . Presentamos la carga máxima de cada parámetro para mostrar la matriz factorial completa de carga límite. Por supuesto, las variables significativas se destacan en negrita y se consideran como principales al formular los nombres de los factores de los estados mentales dominantes. Pasemos a describir los factores determinados.

F1 "La dominación egoísta" es el estado mental más significativo de los encuestados, que refleja la dependencia de los rasgos individuales de la personalidad, es decir, de la motivación interna y cognitiva, del esfuerzo de voluntad, la evaluación del potencial propio, del nivel previsto de la movilización de esfuerzos. Este estado tiene una correlación significativa negativa. Los encuestados demuestran confrontación y un alto nivel de autocontrol en el desempeño de sus funciones oficiales. Los encuestados en los cuales domina el estado mental actual demuestran la actividad interna y cognitiva en su actividad profesional.

F2 "La dominación de confrontación” demuestra la prevalencia de la confrontación en las acciones del encuestado. Tales sujetos tienen la iniciativa, pueden ir a la confrontación, tratan de evitar la búsqueda de otras resoluciones del problema. Su objetivo es evitar la responsabilidad y reevaluar permanentemente y positivamente la situación.

F3 "La dominación de la adquisición" es el estado mental de los encuestados que se esfuerzan por competir a toda costa. Tener un alto nivel de la motivación de competición y la motivación para triunfar sin reevaluar de modo positivo la situación real. El estado mental dominante de tales militares aspira a lograr el resultado deseado a toda costa.
F4 "La dominación pragmática" es el deseo de planificar, resolver problemas y predecir el curso de los acontecimientos. Este estado mental dominante suele estar orientado a buscar la aprobación, el deseo de protegerse a sí mismo, su posición y su punto de vista.

F5 "La domincaión de la evitación" ha combinado los parámetros cuyo contenido refleja el deseo del encuestado de evitar su responsabilidad. Dichos encuestados no muestran ninguna iniciativa y tienen un bajo rendimiento. El objetivo de tales militares es el deseo de evitar cualquier tarea, responsabilidad. Intentan cumplir su servicio militar sin hacer casi nada.

F6 "La dominación afiliativa" refleja el deseo de elogio y reconocimiento. Estos militares toman la iniciativa, se esfuerzan por realizar tareas complejas, buscan constantemente el apoyo social de los demás.

F7 "La dominación profesional" demuestra que el encuestado tiene un estado mental, que está acompañado por el deseo de desempeñar sus funciones de manera responsable. El cumplimiento de deberes se convierte en un culto. Estos encuestados efectuan de modo honesto y correcto todas las tareas, desde las más simples hasta las más complejas.

Constatamos que los otros factores tienen una carga menor a uno y de ellos el mayor factor es 976. Así, hemos determinado siete factores principales que constituyen el $71,651 \%$ de la dispersión. La estructura factorial de los estados mentales dominantes de los militares está determinada por siete factores principales (Fig. 1). 


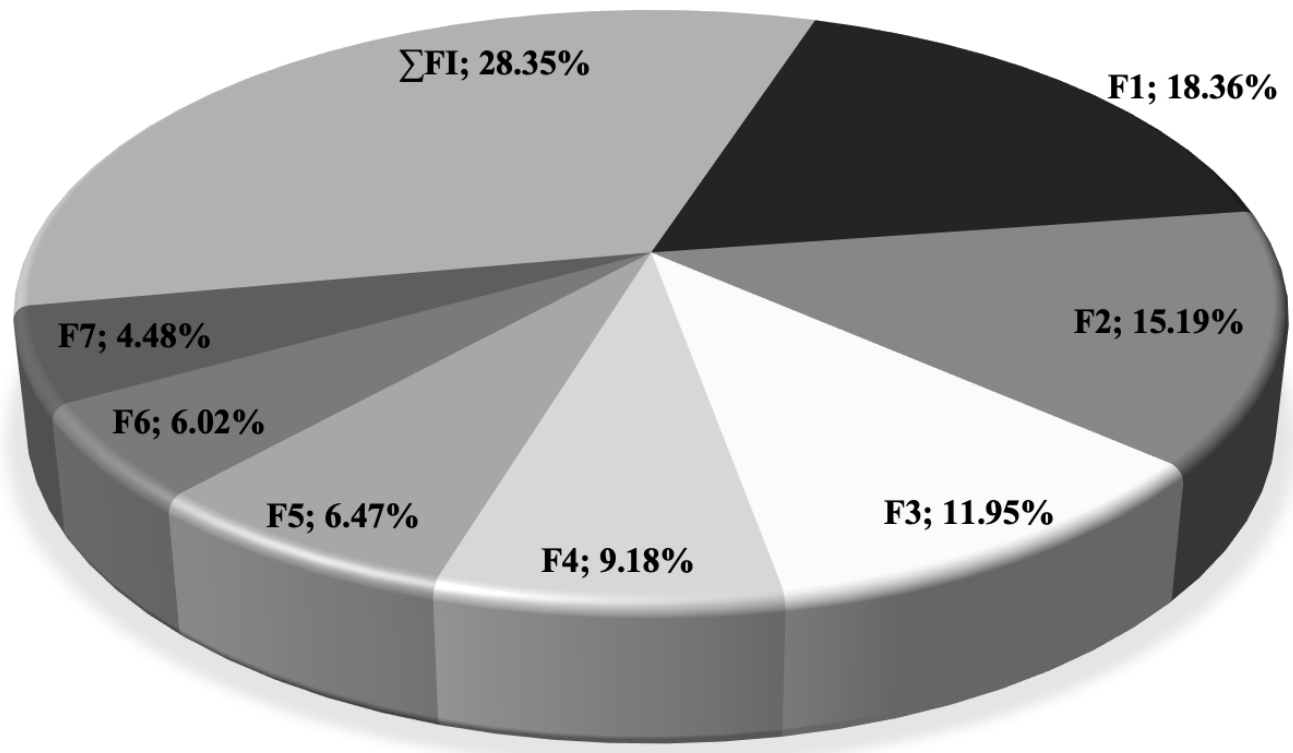

Figura 1. Estructura de los estados mentales dominantes de los militares

Fuente: Elaboración propia, 2021.

La estructura de los estados mentales dominantes de los militares está presentada gráficamente, donde F1 - F7 son los estados mentales dominantes y $\Sigma \mathrm{FI}$ - otros factores con la carga inferior a uno, que es el $28.35 \%$ de la dispersión.
La interdependencia de los factores de estados mentales dominantes de militares. Se ha definido los vínculos más fuertes entre los factores determinados (Tabl. 5).

Tabla 5.

Matriz de correlación de la interdependencia de los factores

\begin{tabular}{rrrrrrrr}
\hline Factor & $\mathrm{F} 1$ & $\mathrm{~F} 2$ & $\mathrm{~F} 3$ & $\mathrm{~F} 4$ & $\mathrm{~F} 5$ & $\mathrm{~F} 6$ & $\mathrm{~F} 7$ \\
\hline F1 & 1.000 & & & $.333^{* *}$ & $-.102^{*}$ & & \\
F2 & & 1.000 & & $-.079^{*}$ & $.220^{* *}$ & & $-.162^{* *}$ \\
F3 & & & 1.000 & $.250^{* *}$ & & $.214^{* *}$ \\
F4 & $.333^{* *}$ & $-.079^{*}$ & $.250^{* *}$ & 1.000 & $-.168^{* *}$ & $.97 *$ & $.140^{* *}$ \\
F5 & $-.102^{*}$ & $.220^{* *}$ & & $-.168^{* *}$ & 1,000 & $-.263^{* *}$ & $-.194^{* *}$ \\
F6 & & & & $.97 *$ & $-.263^{* *}$ & 1.000 & \\
F7 & & $-.162^{* *}$ & $.214^{* *}$ & $.140^{* *}$ & $-.194^{* *}$ & & 1.000 \\
\hline
\end{tabular}

Fuente: Elaboración propia, 2021.

Nota: Método para determinar los factores - Método de componentes principales; Método de conversión - Promax con la normalización de Kaiser; * - fiables con $\mathrm{p} \leq .05 ; * *$ - fiables con $\mathrm{p} \leq .01$.

Los más significativos $(\mathrm{p} \leq .01)$ son los cocientes F1 y F4 (.333), F5 y F6 (-.263), F3 y F4 (.250). F4 tiene la cantidad más grande de las relaciones más significativas con todos los factores F1, F2, F3, F5, F6 y F7. Así, la motivación pragmática es el componente más importante de la organización estructural y funcional de los estados mentales dominantes de militares. Los factores más dependientes de la estructura de los estados dominantes son: F4, F5 y F7.

\section{Discusión}

En la literatura científica no hay muchas investigaciones sobre la psicología militar, la de la motivación, en particular la motivación de los militares, los estudios de los estados mentales motivacionales. Los investigadores han estudiado el problema de los parámetros psicológicos de la motivación de militares (Popovych \& Aleksieieva, 2019). Se determinó empíricamente las relaciones significativas entre los parámetros de la motivación de militares con expectativas sociales ( $\mathrm{p}<.05 ; \mathrm{p}<.01)$ y las orientaciones de sentido de vida $(\mathrm{p}<.05 ; \mathrm{p}<.01)$. En otra investigación se determinó los estados mentales motivacionales de militares (Popovych, 2019). Este estudio confirma nuestros resultados obtenidos e incluso los complementa significativamente. Los estados motivacionales 


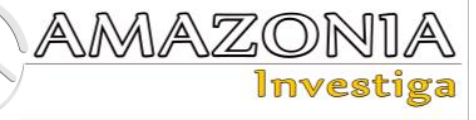

de "ego-motivación", "motivación pragmática" y "motivación afiliativa" son especialmente actuales. En el contexto científico y metodológico, consideramos valiosos los estudios de los estados mentales cognitivos durante la situación simulada de la actividad intelectual de estudiantes (Prokhorov et al., 2015). En otro estudio experimental se definió la estructura factorial, las variables y la interdependencia de los factores de estados mentales de ansiedad de los cadetes del primer año de estudios de la Universidad de seguridad de vida (Popovych et al., 2021b).

En la actividad profesional permanente, los estados mentales motivacionales activos de los militares adquieren estabilidad, consistencia, experimentan una formación particular y el desarrollo, se transforman en los rasgos de personalidad, lo que a su vez afecta las características semánticas de la actividad profesional. Es en esta transformación que, por un lado, reside la capacidad de alcanzar la prosperidad profesional, el apogeo y, por el otro, las frustraciones y deformaciones profesionales. Por lo tanto, la dominación de uno u otro estado mental motivacional afecta ciertamente las características semánticas de la actividad profesional, de servicio (combate) y los resultados de los militares. En particular, F1 "La dominación egoísta" en combinación con F4 "La dominación pragmática" pueden dar el resultado más alto de la actividad profesional. Los estados mentales F5 "La dominación de la evitación" y F6 de "La dominación afiliativa" se oponen uno a otro, lo que es importante para la actividad profesional de militares. Es especialmente importante que los comandantes inmediatos quienes ejercen la gestión táctica, sepan identificar los estados mentales dominantes del personal militar. La identificación de estados mentales es importante cuando se forman los grupos de exploradores, grupos que realizan tareas oficiales (de combate). Esto puede tener la importancia crucial durante el servicio profesional, sin hablar de las actividades de combate de militares.

Se confirma la hipótesis y constatamos que los resultados del estudio de los estados mentales dominantes de militares contienen hechos científicos importantes que deben ponerse en práctica en la formación educativa, profesional y táctica de los militares. Tenemos motivos para afirmar que los resultados del estudio de los estados mentales dominantes de militares ayudarán al personal de mando y los oficiales a resolver problemas de actividad profesional de servicio (combate). La cuestión de la interrelación entre el estado mental dominante concreto de un militar y los resultados de su actividad profesional permanece abierta. Asimismo, nos vemos obligados a reconocer que la identificación de los estados mentales dominantes requiere una formación psicológica profesional de alta calidad, que deben tener el personal de mando y los oficiales.

\section{Conclusiones}

1. Constatamos que los estados mentales dominantes de los militares son una formación mental dinámica compleja que surge durante la realización de tareas, es un indicador particular de la preparación para realizar la tarea. Los estados mentales dominantes están dotados de una función pronóstica hacia el éxito de la tarea. El conjunto propuesto de métodos válidos y métodos de procesamiento de datos estadísticos permitió interpretar cualitativamente los estados mentales dominantes, separar un estado de otro, describir los parámetros del estudio.

2. El análisis factorial ANOVA determinó la estructura de los estados mentales dominantes de los militares, que consta de siete factores principales $(71,651 \%)$. Se descubrió que el componente más importante de la organización estructural y funcional de los estados mentales dominantes de los encuestados es F4 "La dominación pragmática". Este estado tiene más interrelaciones significativas $(\mathrm{p} \leq .01)$ y la correlación F4 y F1 (.333) es la más importante.

3. Se argumentó que la estructura, las variables y la interdependencia de los factores de estados mentales dominantes son componentes importantes para resolver problemas de la actividad profesional, de servicio (combate) de los militares.

4. Los resultados del estudio pueden ser importantes para los jefes de unidades militares, personal de mando, oficiales y directores de escuelas militares.

5. Vemos la perspectiva de la investigación científica en la puesta en práctica de los resultados obtenidos en el sistema de apoyo sociopsicológico de militares en las condiciones de cambios transformadores de la sociedad. La cuestión de la interrelación entre el estado mental dominante particular de un militar y los resultados de la actividad profesional también sigue siendo abierta. 


\section{Referencias bibliográficas}

Arbeláez-Campillo, D. F., Rojas-Bahamón, M. J., \& Arbeláez-Encarnación, T. F. (2019). Apuntes para el debate de las categorías ciudadanía universal, derechos humanos y globalización // Notes for the debate of the categories universal citizenship, human rights and globalization. Cuestiones Políticas, 34(61), 139-161. Recuperado a partir de https://produccioncientificaluz.org/index.php /cuestiones/article/view/30192

Arbeláez-Campillo, D. F., Dudareva, M. A., \& Rojas-Bahamón, M. J. (2019). Las pandemias como factor perturbador del orden geopolítico en el mundo globalizado. Cuestiones Políticas, 36(63), 134-150.

Blynova, O., Kisil, Z., Tkach, T., et al. (2020a). Psychological manifestations of professional marginality of future social welfare professionals. Revista Inclusiones, 7(SI), 218-233.

http://www.revistainclusiones.org/index.php /inclu/article/view/1229

Blynova, O., Moiseienko, V., Los, O., et al. (2020b). Assertiveness as a Factor of Students' Choice of Behavior Strategies in Social Interaction. Revista Inclusiones, 7(4), 259-272.

http://www.revistainclusiones.org/index.php /inclu/article/view/1551

Blynova, O. Ye. \& Kruglov, K. O. (2019) The value of social capital for the psychological well-being of employees. Insight: the psychological dimensions of society, 1, 72-78. DOI: http://doi.org/10.32999/2663970X/2019-1-11

Cheban, Y. V., Chebykin, O. Y., Plokhikh, V. V., et al. (2020). Emotional factor of competitive self-mobilization of professional rowers. Insight: the psychological dimensions of society, $\quad 3$, 28-43. http://doi.org/10.32999/2663-970X/2020-3-2

Center for Systemic Peace. (2017). Global Conflict Trends: Assessing the Qualities of Systemic Peace. New-York: CSP. http://www.systemicpeace.org/conflicttrends .html

Elers, T. (2002). Motivation for Achieving Success and Avoiding Failures. St. Petersburg: Piter. http://um.co.ua/14/145/14-58142.html

Halian, A., Halian, I., Burlakova, I., et al. (2020). Emotional Intelligence in the Structure of Adaptation Process of Future Healthcare Professionals. Revista Inclusiones, 7(3), 447-460.

http://www.revistainclusiones.org/index.php /inclu/article/view/1347
Halian, A., Halian, I., Popovych, I., et al. (2021). Stress Resistance in the Situation of Uncertainty as a Factor of Development of Adaptive Ability of Medical Personnel. BRAIN. Broad Research in Artificial Intelligence and Neuroscience, 12(1), 251-264. https://doi.org/10.18662/brain/12.1/181

Hekhauzen, H. (2003). Motivation and activity. St. Petersburg: Piter. https://altairbook.com/books/770166015motivaciya-i-deyatelnost.html

Herbachevskyi, K. (1990). Psychological diagnostics. Methodology: the level of personality claims. St. Petersburg: Rech. https://hrliga.com/index.php?module=news \&op=view\&id=13493

Hudimova, A. Kh. (2021). Psychological wellbeing and social media users' behavioral online patterns in everyday life and during COVID-19 pandemic. Insight: the psychological dimensions of society, 5, 133-147. https://doi.org/10.32999/2663970X/2021-5-9

Hudimova, A., Popovych, I., Baidyk, V., et al. (2021). The impact of social media on young web users' psychological well-being during the COVID-19 pandemic progression. Revista Amazonia Investiga, 10(39), 50-61. https://doi.org/10.34069/AI/2021.39.03.5

Izard, C. (1991). The Psychology of Emotions (p. 451). New York, NY: Plenum Press. http://dx.doi.org/10.1007/978-1-48990615-1

Khmiliar, O., Popovych, I., Hrys, A., et al. (2020). Spatial Regulation of Personality Behavior in the Conditions of Progression of the COVID-19 Pandemic. Revista Inclusiones, 7(SI), 289-306. http://www.revistainclusiones.org/index.php /inclu/article/view/1760

Klenina, K. V. (2019). Theoretical and methodological analyzing of content characteristics of an individual's perfectionism. Insight: the psychological dimensions of society, 1, 84-89. https://doi.org/10.32999/2663-970X/2019-113

Kononenko, O., Kononenko, A., Stynska, V., et al. (2020). Research of the factor structure of the model of world view settings at a young age. Revista Inclusiones, 7(3), 98-116. http://www.revistainclusiones.org/index.php /inclu/article/view/1618

Kriukova, T. L., \& Kuftiak, Ye. V. (2007). The questionnaire of controlling (the adaptation of the methods WCQ). Journal of an applied psychology specialist, 3(93), 102-112. 


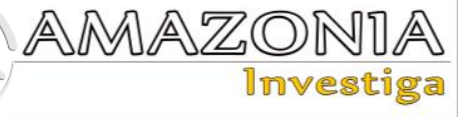

Retrieved from https://www.twirpx.com/file/1656062/

Kuzikova, S., Shcherbak, T., Blynova, O., et al. (2021). Empirical Research of the Hardiness of the Personality in Critical Conditions of Life. Postmodern Openings, 12(2), 57-77. https://doi.org/10.18662/po/12.2/297

Lazarus, R. S., \& Folkman, S. (1984). Stress, appraisal, and coping. New-York: Springer Publishing https://www.worldcat.org/title/stressappraisal-and-coping/oclc/609341596

Ma, F., Shevchenko, R. P., \& Karhina, N. V. (2020). Student you three presentation of psychological well-being: results of content analysis of works. Insight: the psychological dimensions of society, 3, 44-55. https://doi.org/10.32999/2663-970X/2020-33

Marcora, S. M., Staiano, W. \& Manning, V. (2009). Mental fatigue impairs physical performance in humans. Journal of Applied Physiology, 106(3), 857-864. https://doi.org/10.1152/japplphysiol.91324.2 008

Nosov, P. S., Popovych, I. S., Cherniavskyi, V. V., et al. (2020). Automated identification of an operator anticipation on marine transport. Radio Electronics, Computer Science, Control, 3, 158-172. https://doi.org/10.15588/1607-3274-2020-315

Pinkovetskaia, I., Kryukova, L., Arbeláez, D., et al. (2019). Female Entrepreneurship: Types of Economic. Activity. Tarih kultur ve sanat arastirmalari dergisi-journal of history culture and art research, 8(2), 253-265. DOI: http://doi.org/10.7596/taksad.v8i2.2153

Plokhikh V. V. (2021). Assessment of subject's readiness for urgent actions using the variations of sensorimotor response tasks. Insight: the psychological dimensions of society, 5 , 46-65. http://doi.org/10.32999/2663-970X/2021-5-4

Plokhikh, V., Popovych, I., Zavatska, N., et al. (2021). Time Synthesis in Organization of Sensorimotor Action. BRAIN. Broad Research in Artificial Intelligence and Neuroscience, 12(4), 164-188. https://doi.org/10.18662/brain/12.4/243

Popovych, I., Arbeláez-Campillo, D. F., RojasBahamón, M. J., et al. (2021a). Time perspective in the professional activity of specialists of economic sphere. Cuestiones Políticas, 39(69), 424-445 https://doi.org/10.46398/cuestpol.3969.27

Popovych, I., Chervinskyi, A., Kazibekova, V., et al. (2021b). Empirical research of the typology of social expectations of the personality. Amazonia Investiga, 10(43), 112-122.

https://doi.org/10.34069/AI/2021.43.07.11

Popovych, I., Halian, O., Bokhonkova, Yu., et al. (2020a). Research of the Motivational Mental States of Future Bachelors of the Faculty of Physical Education and Sports in the Educational Process. Revista Inclusiones, 7(4), 159-178. http://www.revistainclusiones.org/index.php /inclu/article/view/1545

Popovych, I., Shevchenko, A., Galvez, L. M., et al. (2021c). Research of the relationship between social desirability and value orientations of adolescents. Revista Notas Históricas y Geográficas, 26(1), 241-268. https://www.revistanotashistoricasygeografi cas.cl/index.php/nhyg/article/view/339

Popovych, I. S. \& Aleksieieva, M. I. (2019). Study of psychological content parameters of servicemen' motivation. Fundamental and applied researches in practice of leading scientific schools, 34(4), 26-33. https://doi.org/10.33531/farplss.2019.4.4

Popovych, I. S. (2019). Research of motivational mental states of servicemen. Theoretical and applied problems of psychology, 3(50), 158-173.

https://rep.ksma.ks.ua/bitstream/123456789/ 346/1/nosov_teoretiko_method.pdf

Popovych, I. S. (2014). Social expectations in primary school age. Proceedings of the 2 nd International Academic Congress "Fundamental Studies in America, Europe, Asia and Africa", 2, 176-180. http://ekhsuir.kspu.edu/handle/123456789/3 306

Popovych, I., Tkach, T., Sirko, R., et al. (2020b). Research on Mental States of Anxiety of First-Year Cadets of the University of Life Safety. Revista Inclusiones, 7(SI), 264-278. http://www.revistainclusiones.org/index.php /inclu/article/view/1233

Prokhorov, A. O., Yusupov, M. G. \& Plokhikh, V. V. (2015). Cognitive States in the Process of Students' Intellectual Activity. The New Educational Review, 41(3), 263-274.

https://doi.org/10.15804/tner.2015.41.3.21

Semenov, O., Oleshko, P., Tsymbal, S., et al. (2021). Research of social expectations of university students in the dimensions of psychological well-being. Revista Eduweb, 15(3), 124-138. https://doi.org/10.46502/issn.18567576/2021.15.03.10

Shevchenko, A. V. (2019). Research on the correlation between social desirability and value orientations in adolescence. Insight: the 
psychological dimensions of society, 1, 90-94. https://doi.org/10.32999/2663970X/2019-1-14

Shevchenko, R., Cherniavskyi, V., Zinchenko, S., et al. (2020a). Research of psychophysiological features of response to stress situations by future sailors. Revista Inclusiones, $\quad 7(\mathrm{SI}), \quad$ 566-579. http://www.revistainclusiones.org/index.php /inclu/article/view/1780

Tataryn, I., Komissarchuk, Y., Dmytryk, Y., et al. (2021). Features of detection and obtaining evidence of war crimes committed in the context of international armed conflict. Cuestiones Políticas, 39(69), 74-90. https://doi.org/10.46398/cuestpol.3969.04

World Medical Association Declaration of Helsinki. (2013). Ethical principles for medical research involving human subjects, 310(20),

2191-4. https://doi.org/10.1001/jama.2013.281053

Zinchenko, S. M., Ben, A. P., Nosov, P. S., et al. (2020). Improving the accuracy and reliability of automatic vessel moution control system. Radio Electronics, Computer Science, Control, 2, 183-195. https://doi.org/10.15588/1607-3274-2020-219

Zinchenko, S., Nosov, P., Mateichuk, V., et al. (2019). Automatic collision avoidance system with many targets, including maneuvering ones. Bulletin of University of Karaganda, 96(4), 69-79. https://physicsvestnik.ksu.kz/apart/2019-96-4/8.pdf 